\title{
Análisis de los modelos de negocio en internet: Situación actual en el subsector de transporte intermunicipal de pasajeros en el área metropolitana de Bucaramanga
}

Analysis of internet business models: the current situation in the intermunicipal passenger transport subsector in the metropolitan area of Bucaramanga

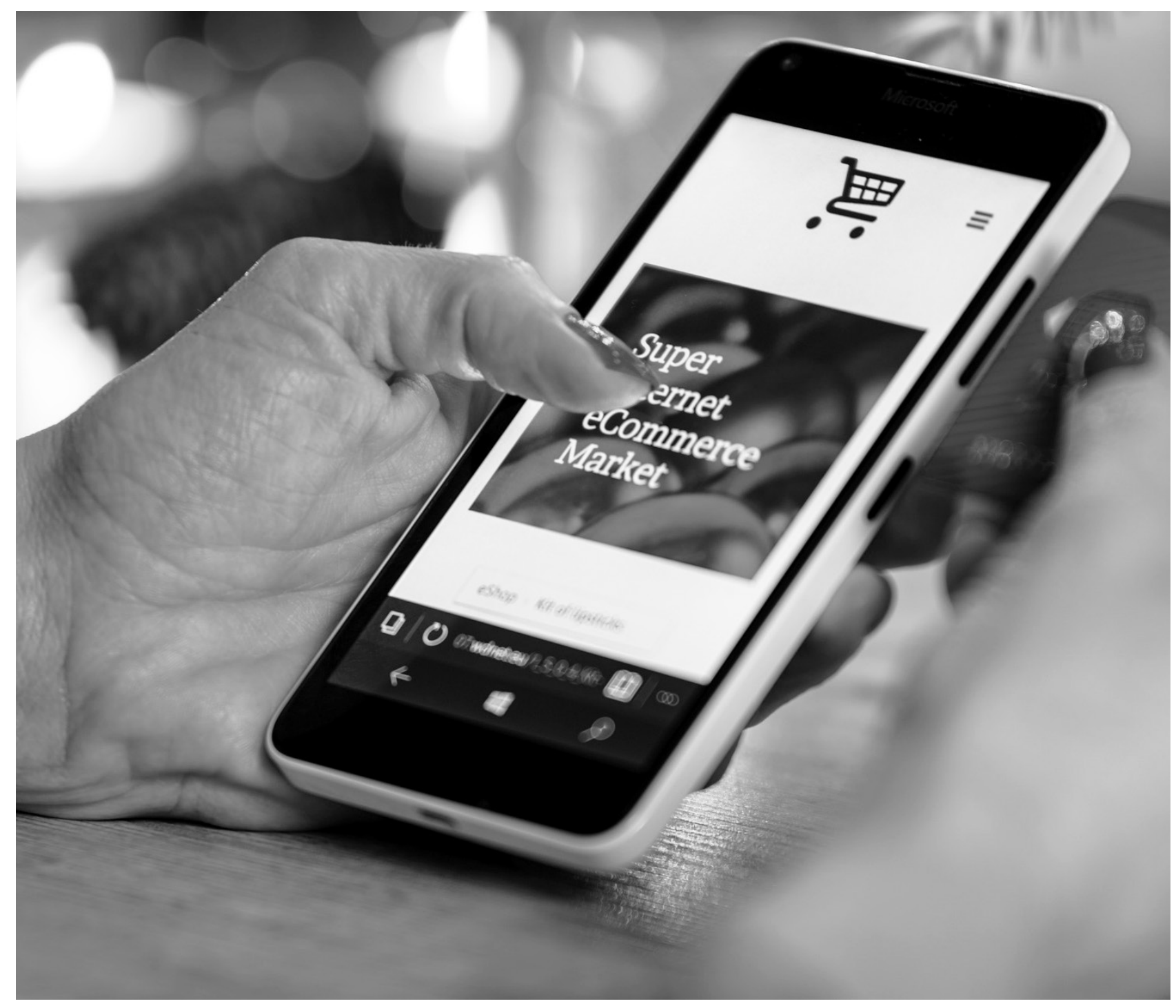




\title{
Análisis de los modelos de negocio en internet: Situación actual en el subsector de transporte intermunicipal de pasajeros en el área metropolitana de Bucaramanga ${ }^{1}$ Analysis of internet business models: the current situation in the intermunicipal passenger transport subsector in the metropolitan area of Bucaramanga
}

\author{
Katherine Julieth Sierra Suárez², Jessica Tatiana Rey Ordoñez², \\ Angie Patricia Barrios Machado ${ }^{4}$
}

Artículo recibido en febrero 25 de 2019; artículo aceptado en mayo 13 de 2019.

Este artículo puede compartirse bajo la Licencia Creative Commons Atribución-NoComercial-Compartirlgual 4.0 Internacional y se referencia usando el siguiente formato: Sierra; S. K., Rey, O. J. \& Barrios, M. A. (2019). Análisis de los modelos de negocio en internet: Situación actual en el subsector de transporte intermunicipal de pasajeros en el área metropolitana de Bucaramanga.

I+D Revista de Investigaciones, 14 (2), 102-112. DOI: https://doi.org/10.33304/revinv.v14n2-2019009

\begin{abstract}
Resumen
Este artículo analizó los modelos de negocio en internet y la ventaja competitiva de las empresas del subsector transporte intermunicipal de pasajeros en el área metropolitana de Bucaramanga. El objetivo principal es conocer la posición actual de cada modelo según la teoría de Faisal Hoque, y así brindar tácticas de mejora a las organizaciones. El estudio de caso se realizó con tres empresas del subsector que brindaron información de su negocio presencial y en internet. Realizando un análisis cuantitativo de las variables y utilizando técnicas de investigación tales como entrevistas, encuestas y listas de chequeo, se evaluaron los modelos de negocio electrónicos, y se posicionó a las empresas en las categorías brochureware y e-commerce. Por último, se usó la matriz DOFA para generar tácticas de mejora que ayuden a fortalecer la operatividad y la comunicación de cada empresa a lo largo de su cadena de suministro, desde su página web.
\end{abstract}

Palabras clave: Modelo de negocio en internet, sector transporte, ventaja competitiva.

\footnotetext{
Abstract

This paper analyses the internet business models and the competitive advantage of the companies of the

1. Artículo de investigación empírico, alcance descriptivo, diseño no experimental de corte transversal, enfoque cuantitativo, resultado de un proyecto de investigación culminado, perteneciente a la Facultad de Ingenierías, subárea de Ingeniería Industrial y financiado por la Universidad de Investigación y Desarrollo (UDI), de la ciudad de Bucaramanga, Santander (Colombia). Dirección: calle 9 n. ${ }^{\circ} 23-55$, PBX: 6352525. Fecha de inicio: 6 de marzo de 2018. Fecha de terminación: 10 de diciembre de 2018.

2. Magíster en Ingeniería de la Universidad Industrial de Santander, docente de la Escuela de Estudios Industriales y Empresariales, grupo de investigación Finance \& Management, Universidad Industrial de Santander. Dirección: carrera 27 n. 9, ciudad universitaria, Bucaramanga (Colombia). ORCID ID: https://orcid.org/0000-0003-1429-6060. Correo electrónico institucional: katherine.sierra@saber.uis.edu.co

3. Facultad de Ingeniería Industrial, vinculada a la Universidad de Investigación y Desarrollo. Dirección: calle 9 n.` 23-55, PBX: 6352525 Bucaramanga (Colombia). ORCID ID: https://orcid.org/0000-0001-6649-7935. Correo electrónico institucional: jrey4@udi.edu.co

4. Facultad de Ingeniería Industrial, vinculada a la Universidad de Investigación y Desarrollo. Dirección: calle 9 n. ${ }^{\circ} 23-55$, PBX: 6352525 Bucaramanga (Colombia). ORCID ID: https://orcid.org/0000-0002-4350-1097. Correo electrónico institucional: abarrios@udi.edu.co
} 
Katherine Julieth Sierra Suárez, Jessica Tatiana Rey Ordoñez, Angie Patricia Barrios Machado

Análisis de los modelos de negocio en internet: Situación actual en el subsector de transporte intermunicipal de pasajeros en el área

metropolitana de Bucaramanga.

intermunicipal passenger transport subsector in the metropolitan area of Bucaramanga. The main objective is to identify the current position of each business model, according to Faisal Hoque's theory and then provide improvement tactics that add value to their organizations. The case study was carried out with three companies of the subsector, which provided information about their business model on-site and online. By performing a quantitative analysis of the variables and using research techniques such as interviews, surveys, and checklists, electronic business models were evaluated, and the companies were positioned in the Brochureware and E-commerce category. Finally, the SWOT matrix was used to generate improvement tactics that help strengthen the operation and communication of each company throughout its supply chain from their websites.

Keywords: Internet business model, transportation sector, competitive advantage.

\section{Introducción}

Gracias a la globalización, las personas utilizan el internet como una herramienta de comunicación con individuos y empresas. Esto permite interacciones que van desde los intercambios de información hasta las transacciones económicas. Por tal motivo, a finales del siglo XX, y como resultado de la evolución de los modelos de negocios tradicionales, las personas y las organizaciones se interesaron en un nuevo modelo de negocio llamado comercio electrónico o e-commerce (Díaz, 2014; Khurana, 2011). Este se entiende como una nueva economía digital que fundamenta sus estrategias y operaciones comerciales en el valor agregado que se da a los clientes por medio de internet.

Los estudios internacionales indican que el concepto de comercio electrónico comenzó a fortalecerse en Europa a principios del siglo XXI (Martínez Rodríguez, 2015; Sánchez \& Sandulli, 2007), teniendo como principal exponente a Hoque (2000), quien logró posicionar los modelos de negocio electrónico según su nivel de integración de actividades relacionadas con la creación de valor en internet. Sin embargo, en América Latina se tienen pocos estudios sobre el tema (Botello Peñaloza, Pedraza Avella, \& Contreras Pacheco, 2015; Morales Campos \& Prieto Daza, 2017). Debido a esto, las empresas colombianas se han visto forzadas a mejorar su modelo de negocios electrónico en forma empírica, a través de técnicas de ensayo y error.

En la actualidad, en el área metropolitana de Bucaramanga (AMB) se encuentran ubicadas 166 empresas que pertenecen al subsector transporte de pasajeros (Cámara de Comercio de Bucaramanga, 2018). Estas movilizaron 2.489 .653 pasajeros en el año 2018 (Cámara Comercio de Bucaramanga, 2019), en comparación con el transporte aéreo, que movilizó 1.595.907 pasajeros en el mismo año (Cámara de Comercio de Bucaramanga, 2019). Las cifras son positivas, pues muestran que el transporte terrestre sigue siendo la primera opción de desplazamiento para los habitantes del AMB. No obstante, las empresas de este sector no han cambiado en los últimos años, y continúan enfocándose en estrategias de negocio tradicionales. Esto muestra la necesidad de buscar nuevas estrategias de negocio que generen una ventaja competitiva y añadan valor a sus servicios, de modo que logren la fidelización de sus clientes actuales y la captación de nuevos usuarios. Estas estrategias de mejora deben estar alineadas con la evolución económica y tecnológica del país.

Por lo anterior, las autoras de este trabajo realizaron un análisis de los modelos de negocio en internet de las empresas del subsector transporte intermunicipal de pasajeros en el área metropolitana de Bucaramanga, con el objeto de sentar un punto de partida que permita posicionar de forma asertiva a las empresas del estudio de caso. Ello, por medio de un estudio cuantitativo y descriptivo de las variables, con el fin de determinar cuáles serían las tácticas de mejora más adecuadas para aumentar la ventaja competitiva de cada empresa en el mercado, de modo que lleguen a convertirse en empresas e-bussines (empresas que centran su trabajo en la creación de valor a través de internet, mediante el cual crean alianzas estratégicas con proveedores y se integran con clientes).

\section{Metodología}

\section{Tipo de estudio}

Para el desarrollo de la investigación, se implementó un enfoque cuantitativo mediante el análisis de datos numéricos obtenidos de las entrevistas, encuestas y listas de chequeo implementadas. El estudio tuvo un diseño no experimental de corte transversal; cuenta con un alcance descriptivo y con una unidad de estudio de tres empresas. Las variables estudiadas fueron modelo de negocio electrónico (Alfonzo \& Mariño, 2013; Campdesuñer, De Miguel Guzmán \& Almaguer, 2012) y ventaja competitiva (Ma, 1999; Porter, 1980). 


\section{Participantes}

La población en estudio consiste en las 166 empresas del subsector transporte de pasajeros (Cámara de Comercio de Bucaramanga, 2018). La muestra se definió con los siguientes parámetros:

- Marco de muestreo: Listado de 24 empresas que se encuentran en operación en el Terminal de Transportes de Bucaramanga (2019).

- Unidad de análisis: Empresa del subsector transporte de pasajeros que opera en el Terminal de Bucaramanga.

- Muestreo: Se eligió muestreo por conveniencia, debido a que el estudio requería información de documentos internos de la empresa, acceso a su página web e intranet, información primaria de proveedores y empleados. Estas condiciones fueron expuestas ante las empresas que pertenecían al marco muestral, y tres de ellas aceptaron hacer parte del estudio.

En este estudio se determinó una muestra de tres empresas ubicadas en el área metropolitana de Bucaramanga: Cootransmagdalena, Copetrán y Cotaxi. De estas se pudo obtener información de 18 de sus trabajadores, de sus 11 proveedores y de una muestra estratificada de 245 usuarios de las tres empresas.

\section{Materiales e instrumentos}

En la investigación se utilizó observación natural y herramientas de medición, tales como entrevistas, encuestas, listas de chequeo, método de factores críticos de éxito (FCE) (Alonso Ferreras, 2009) y la matriz DOFA. La tabulación y análisis de la información adquirida en el trabajo de campo se registró mediante el programa Microsoft Excel ${ }^{\circledast}$ e IBM SPSS Statistics $23^{\circledR}$.

Mediante visitas de campo, y utilizando la observación natural, se hizo un recorrido de reconocimiento por las instalaciones de las empresas del caso de estudio y las instalaciones del Terminal de Transporte de Bucaramanga. En cuanto a las encuestas, se aplicaron tres formularios: la primera encuesta fue aplicada a trabajadores, e incluyó 30 preguntas enfocadas a medir su conocimiento de la planeación estratégica de la empresa y el uso que le daba a la página web. La segunda encuesta fue aplicada a proveedores y buscaba medir la relación de estos con la empresa, a través de la página web. La tercera y última encuesta se componía de 14 preguntas dirigidas a los clientes, para medir sus características geográficas, sociodemográficas, hábitos de consumo y uso del servicio electrónico ofrecido por las empresas, para identificar las fortalezas y debilidades de la empresa desde su punto de vista. También se aplicaron tres entrevistas semiestructuradas con cada uno de los gerentes de las tres empresas, y por último se utilizaron dos listas de chequeo para la medición y diagnóstico de:

- Planeación estratégica de las empresas del estudio de caso de forma física y digital, conformada por tres niveles de planeación y 20 ítems de evaluación.

- Medición cuantitativa del diseño de las páginas web corporativas, conformada por diez criterios principales y 43 subcriterios.

\section{Matriz DOFA}

Gürel y Tat (2017) realizaron recientemente una revisión teórica de la matriz DOFA, y determinaron que es una valiosa técnica para planear y tomar decisiones. Además, concluyen que, a pesar de sus limitaciones, el examen cualitativo de los factores internos y externos puede ser el comienzo para un análisis en profundidad de la planificación. Debido a esto, se usa la matriz DOFA como una herramienta de análisis para las variables internas y externas de las empresas.

Teniendo en cuenta que las tres cooperativas pertenecen al mismo subsector y están localizadas en la misma área geográfica, en el análisis externo de las tres empresas se tomaron las mismas oportunidades y amenazas, y a nivel interno se escogieron las fortalezas y las debilidades más relevantes de cada cooperativa.

La relevancia de cada fortaleza, oportunidad, debilidad y amenaza se eligió a criterio de las autoras del proyecto, quienes calificaron de 1 a 5 cada cruce de variable. Al realizar la sumatoria de estas, se seleccionaron las dos variables con mayor puntuación, y así se crearon las estrategias FO, FA, DO, DA. Estas son la base de las acciones de mejora para cada organización.

\section{Método de factores críticos de éxito (FCE)}

Se usa el método de factores críticos de éxito (FCE), que permite identificar los puntos clave, el número de aspectos, áreas, ámbitos, variables y condiciones que, al ser evaluados, generan un resultado. Si este es satisfactorio, se garantiza un rendimiento competitivo y de éxito de la empresa. Las autoras del proyecto toman y adecúan al subsector transporte de pasajeros el instrumento expuesto por Alonso Ferreras (2009), quien evaluó los FCE en el sector turismo, mediante un cuadro de mando y la metodología DELPHI. 
Katherine Julieth Sierra Suárez, Jessica Tatiana Rey Ordoñez, Angie Patricia Barrios Machado

Análisis de los modelos de negocio en internet: Situación actual en el subsector de transporte intermunicipal de pasajeros en el área

metropolitana de Bucaramanga.

Para lograr la evaluación de la ventaja competitiva de las empresas objeto de estudio, se utilizó la matriz de comparación pareada, para contrastar, evaluar y asignar prioridades a los 24 FCE. Esto, con la ayuda de un experto interno de cada organización y un experto externo especialista en el tema de transporte. Teniendo en cuenta la sumatoria horizontal de los FCE en la matriz de comparación pareada y la valoración del FCE, se tomarán los valores mayores a trece (13) y con una calificación superior o igual a cuatro (4) como factores críticos de éxito en las empresas objeto de estudio. Los FCE seleccionados se presentan en la Tabla 1.

Tabla 1

Factores críticos de éxito para empresas objeto de estudio

\begin{tabular}{|c|c|c|}
\hline Dimensión crítica & $\begin{array}{l}\text { N. } .^{\circ} \\
\text { FCE }\end{array}$ & $\begin{array}{l}\text { Factor crítico de éxito } \\
\text { (FCE) }\end{array}$ \\
\hline $\begin{array}{l}\text { Dirección y gestión } \\
\text { del destino }\end{array}$ & F7 & $\begin{array}{l}\text { Gestión de los recursos } \\
\text { humanos del destino }\end{array}$ \\
\hline \multirow{3}{*}{$\begin{array}{l}\text { Recursos, } \\
\text { infraestructura, } \\
\text { servicios de apoyo y } \\
\text { complementarios }\end{array}$} & F9 & Recursos humanos \\
\hline & F10 & $\begin{array}{l}\text { Facilidad de acceso } \\
\text { (accesibilidad) }\end{array}$ \\
\hline & $\mathrm{F} 12$ & $\begin{array}{l}\text { Infraestructuras } \\
\text { generales }\end{array}$ \\
\hline $\begin{array}{l}\text { Desarrollo } \\
\text { sostenible }\end{array}$ & F14 & Crecimiento económico \\
\hline $\begin{array}{l}\text { Agentes implicados } \\
\text { (stakeholders) }\end{array}$ & F16 & $\begin{array}{l}\text { Agentes implicados } \\
\text { locales (prestadores de } \\
\text { los servicios de } \\
\text { transporte de pasajeros) }\end{array}$ \\
\hline \multirow{3}{*}{$\begin{array}{l}\text { Cliente (demanda } \\
\text { de pasajes) }\end{array}$} & F17 & Satisfacción del cliente \\
\hline & F18 & $\begin{array}{l}\text { Fidelización y } \\
\text { seguimiento posterior } \\
\text { del cliente }\end{array}$ \\
\hline & F19 & Captación de clientes \\
\hline $\begin{array}{l}\text { Condiciones del } \\
\text { entorno: } \\
\text { internacional, } \\
\text { nacional y sectorial }\end{array}$ & $\mathrm{F} 24$ & $\begin{array}{l}\text { Condiciones del } \\
\text { entorno nacional y } \\
\text { sectorial }\end{array}$ \\
\hline
\end{tabular}

Fuente: Autores.

\section{Procedimiento}

La investigación se realizó en tres etapas: 1) diagnóstico inicial de la planificación estratégica; 2) caracterización de las páginas web y posición actual de los modelos de negocio en internet; y 3) generación de tácticas de mejora para los modelos de negocio en internet.

\section{Diagnóstico inicial de la planificación estratégica}

La planeación estratégica traza los objetivos y las estrategias que implementará cada organización en un corto, mediano o largo plazo, lo que marca el camino que se debe recorrer para el logro de sus metas corporativas; y además brinda las directrices en la toma de decisiones. Por tal motivo, la planeación estratégica se reconoce como un bien invaluable que todas las partes interesadas deben conocer. Debido a esto, es necesario que las organizaciones cuenten con un documento físico o digital que soporte y garantice que todos los niveles de la organización conocerán los pilares, objetivos, misión y visión de la empresa y su área.

El instrumento aplicado para la medición y diagnóstico de la planeación estratégica de las empresas objeto de estudio fue una lista de chequeo cuyo diseño incluyó tres secciones: planeación estratégica corporativa, planeación táctica y planeación operativa. Para su diligenciamiento se tomaron como referencia los documentos físicos internos y la información de la página web de cada cooperativa.

\section{Caracterización de las páginas web y posición actual de los modelos de negocio en internet}

Para el desarrollo de esta sección, se tuvo en cuenta una valoración cualitativa y cuantitativa de las variables. Inicialmente se utilizó una lista de chequeo para revisar si las 24 empresas del subsector estudiado que están en funcionamiento en la Terminal de Transporte de Bucaramanga tienen página web corporativa y venta de tiquetes en línea. En segundo lugar, se identificó el tipo de comercio electrónico que realizan estas empresas, que puede ser:

- Comercio electrónico B2B o business to business: Son las transacciones que se realizan de un negocio a otro negocio. Los dos deben operar desde la web; en este modelo no interviene el consumidor.

- Comercio electrónico B2C o business to consumer: Transacciones que se realizan entre un negocio electrónico y una persona particular o consumidor.

- Comercio electrónico B2E o business to employee: Se centra en las transacciones entre la organización y sus empleados; es decir, las ofertas o beneficios que tendrían los trabajadores en el momento que necesiten adquirir un producto o servicio de la compañía en la que trabajan. - Comercio electrónico C2C o consumer to consumer: Este tipo de transacciones se da cuando un consumidor decide vender un artículo ya usado por él mismo, por medio de internet, a otro consumidor que pueda estar interesado en adquirirlo.

- Comercio electrónico G2C o goverment to consumer: Se refiere a la facilidad que brinda el gobierno a las personas, para realizar trámites a través de una plataforma digital de la entidad gubernamental.

En tercer lugar, se logró ubicar a cada una de las empresas 
en una de las cuatro categorías de modelo de negocios expuestas por Faisal Hoque (2000): 1) brochureware: organizaciones con presencia en la red, mediante una página web corporativa básica que brinda a los usuarios información de la organización y del producto o servicio que ofrece. 2) E-commerce: compañías que no solo dan a conocer información de sus productos y servicios, sino que además facilitan un medio para la compra y venta de estos. 3) E-business: empresas que centran su trabajo en la creación de valor mediante la armonía de la cadena de suministro. Así se crean alianzas estratégicas con los proveedores y se integran a los clientes en todo el sistema, todo esto a través de internet. 4) E-enterprise: conocidas como empresas electrónicas, rigen su modelo de negocio totalmente en la red; es decir, son empresas virtuales en todos sus aspectos.

Por último, en la medición cuantitativa del diseño de las páginas web corporativas, se aplicó una lista de chequeo conformada por 10 criterios principales y 43 subcriterios. Estas listas fueron diligenciadas con la ayuda de un experto interno de cada organización y de cuatro expertos externos, todos profesionales en el tema de modelos de negocio en internet.

\section{Tácticas de mejora para los modelos de negocios en internet}

Para el desarrollo de esta sección, se tuvo en cuenta la valoración de las encuestas aplicadas a los clientes, trabajadores y proveedores de cada una de las empresas del estudio de caso. Además, se evaluó la ventaja competitiva de las tres empresas, por medio del método factores críticos de éxito (Alonso Ferreras, 2009). Los factores fueron priorizados mediante una matriz de comparación pareada, que fue realizada por un experto en el sector transporte. Con base en esta herramienta, se pudo medir la ventaja competitiva de las empresas objeto de estudio. Por último, se aplicó la matriz DOFA, como una herramienta de análisis para las variables internas y externas de las empresas, con el objeto crear estrategias FO, FA, DO, DA, para cada una de estas empresas como tácticas de mejora para el modelo de negocio en internet. Es apropiado mencionar que debido a que las tres cooperativas pertenecen al mismo subsector y están localizadas en la misma área geográfica, en el análisis externo se tomaron las mismas oportunidades y amenazas.

\section{Resultados}

Los resultados de la aplicación de cada etapa se presentan a continuación.

\section{Diagnóstico inicial de la planificación estratégica}

Como resultado general se obtuvo que el nivel de cumplimiento de la planeación estratégica de las empresas objeto de estudio se encuentra entre un $40 \%$ y $50 \%$ de forma física, y entre un $26 \%$ y $32 \%$ de forma virtual. Tiene sus principales falencias en la planeación táctica y operativa de cada empresa; por otro lado, en las páginas web de cada cooperativa no se pueden apreciar las estrategias actuales ni la ventaja competitiva que maneja cada empresa para sus modelos de negocio en internet. Como resultado, los usuarios no logran conocer los beneficios que les brinda cada empresa por este medio.

\section{Caracterización de las páginas web y posición actual de los modelos de negocio en internet}

Al analizar las 24 empresas del subsector transporte de pasajeros, se observa que el $79 \%$ de estas empresas cuentan con página web corporativa, y el 54 \% cuenta con venta de tiquetes en línea. Esto permite que sus clientes externos tengan la posibilidad de adquirir de forma virtual los servicios ofrecidos por la empresa.

Por otro lado, con base en las teorías existentes, las autoras logran caracterizar el tipo de comercio electrónico que usan las tres cooperativas estudiadas: todas permiten transacciones de la empresa con sus clientes por medio de la página web corporativa (B2C) y también la comunicación interna de la organización con sus colaboradores (B2E).

Por otra parte, usando el modelo de Hoque (2000) se logra posicionar a la empresa Cootransmagdalena Ltda. en la categoría de brochureware. Esto se debe a que, si bien es cierto que la organización cuenta con una página web bien estructurada y, por medio de esta, facilita la compra y venta de tiquetes en línea, está aún no es reconocida por la mayoría de sus usuarios; por tanto, su estrategia de promoción virtual aún no está siendo percibida por el segmento de mercado. Por su parte, la empresa Copetrán Ltda. se posiciona en la categoría de e-commerce, puesto que su modelo de negocio en internet se encuentra fuertemente estructurado y obtiene un nivel medio en el reconocimiento de su página web por parte de sus clientes, quienes han llegado a utilizar la página web o la aplicación móvil para comprar su tiquete. De la misma forma, la empresa Cotaxi Ltda. se posiciona en la categoría e-commerce. Esta empresa cuenta con un bajo reconocimiento de su página web, pero en las encuestas aplicadas a los clientes se visualizó la compra de tiquetes de forma virtual o por domicilio, lo que quiere decir que su estrategia de promoción virtual está empezando a 
Katherine Julieth Sierra Suárez, Jessica Tatiana Rey Ordoñez, Angie Patricia Barrios Machado

Análisis de los modelos de negocio en internet: Situación actual en el subsector de transporte intermunicipal de pasajeros en el área

metropolitana de Bucaramanga.

funcionar, y sus clientes están comenzando a usar sus canales de venta en línea.

Adicionalmente, se realizó una evaluación cuantitativa del modelo de negocio en internet usando una lista de chequeo diligenciada por expertos internos y externos. Gracias a ello, se logra concluir que Copetrán cuenta con el mejor diseño web, con un total de 440 puntos. Cotaxi se encuentra en un segundo lugar, con un total de 391 puntos, y en el tercer lugar está Cootransmagdalena, con un total de 369 puntos. En esta evaluación de la calidad de las tres páginas web se encuentra que las principales falencias de las empresas residen en:

- Las empresas Cootransmagdalena y Cotaxi necesitan la adquisición de su propia plataforma de venta virtual, puesto que no existe ventaja comparativa en su canal de ventas en línea.

- Falta de diferenciación e innovación en la estructura y diseño de la página web corporativa.

- Falta de interactividad en tiempo real con los usuarios.

- Falta de una sección de comunicación social guiada a brindar información oportuna al usuario del sector o la empresa.

- Falta de una sección de noticias que permita ver el enfoque de responsabilidad social que tiene cada empresa.

- Falta de herramientas digitales en las páginas web que faciliten visualizar las estrategias de marketing digital en temas de precios y promoción de los servicios ofrecidos por las cooperativas.

- El nivel de seguridad y confidencialidad de la página web de las empresas Cootransmagdalena y Cotaxi es bajo y poco confiable para una transacción bancaria.

- El sitio web de las tres empresas no logra plasmar las estrategias de fidelización para los clientes; son muy planas y poco atractivas en temas de cambios internos y beneficios para el usuario.

- Las páginas web solo están centradas en brindar información de la empresa y servir como un medio transaccional a la hora de adquirir el tiquete de forma electrónica, pero no buscan conocer a su cliente y sus necesidades. Esto es una barrera para satisfacer las necesidades del usuario y mejorar las estrategias del modelo de negocio en internet.

\section{Tácticas de mejora para cada una de las empresas del estudio de caso}

En primer lugar, se analizan los resultados de las encuestas que fueron aplicadas a clientes, empleados y proveedores:

\section{Encuestas para clientes}

- Las tres empresas tienen una gran acogida por la población bumanguesa, dado que cuentan con un reconocimiento de varios años en el sector. Sus principales fuentes de publicidad son las referencias y la visualización de los vehículos de la flota empresarial.

- El perfil del cliente lo constituyen hombres y mujeres entre los 18 y 40 años, población estudiantil y trabajadora que viaja principalmente por motivos de negocios, familiares, educativos o turísticos; la mayoría con una frecuencia de una o más veces por semana o al mes.

- El nivel de conocimiento de las páginas web corporativas es bajo, lo que presenta principalmente barreras en el acceso a internet y la incertidumbre en la información y el proceso de compra. De esto, se obtiene como resultado que los usuarios prefieren comprar por ventanilla y no por la página web.

\section{Encuestas para los trabajadores:}

- Las empresas están trabajando en la fidelización del cliente interno, lo que hace que conozcan y se enamoren de la empresa por medio de capacitaciones, juntas y manuales que hablen sobre la planeación estratégica corporativa. Como resultado, obtienen personal con varios años de experiencia en los cargos analizados.

- Para los trabajadores, las páginas web corporativas cuentan con una interfaz adecuada. Este modelo de negocio tiene como objetivo principal el aumento de las ventas; $y$ como principales falencias, las fallas técnicas de las páginas web y la pérdida de tiquetes.

- Las empresas centran sus esfuerzos en crear una ventaja competitiva centrada en la diferenciación de costos y el reconocimiento en el ámbito nacional.

\section{Encuestas para proveedores:}

- Las empresas tienen un alto nivel de fidelización con sus proveedores, quienes en su mayoría tienen conocimiento de la página web de las cooperativas; pero estas no cuentan con un espacio u enlace para comunicarse con ellos por medio del sitio web.

- Los principales medios de comunicación con los proveedores es el correo electrónico, el sistema financiero-contable y por vía telefónica. Las empresas han establecido un tiempo de respuesta a proveedores de 1 a 8 horas.

En segundo lugar, se usa el método de factores críticos de éxito para evaluar la ventaja competitiva de las tres 
Katherine Julieth Sierra Suárez, Jessica Tatiana Rey Ordoñez, Angie Patricia Barrios Machado Análisis de los modelos de negocio en internet: Situación actual en el subsector de transporte intermunicipal de pasajeros en el área metropolitana de Bucaramanga.

empresas. Según estas evaluaciones, se puede concluir que, con un total de 84 puntos, Copetrán cuenta con la mejor ventaja competitiva del mercado, seguida por Cotaxi, con 73 puntos; por último, Cootransmagdalena, con un total de 70 puntos.

En tercer lugar, y usando los resultados anteriores, se proponen tácticas de mejora para las tres empresas estudiadas.

\section{Cootransmagdalena}

- Aprovechar su posicionamiento y reconocimiento para subir los precios, con miras a ofrecer mejores servicios en sus rutas más reconocidas.

- Intensificar las estrategias web de promoción para los usuarios frecuentes de estas rutas, tales como paquetes redondos, acumulación de millas, entre otras.

- Adquirir su propia plataforma de compra y venta de tiquetes en línea, para distinguirse de su competencia, dado que tienen una estructura web similar.

- Dar a conocer a sus usuarios frecuentes las estrategias de precios dirigidas a descuento para clientes que compren por la página web.

- Crear estrategias combinadas de fidelización para los clientes frecuentes y los mejores vendedores de la empresa.

- Crear canales de comunicación sincrónica, como el chat en línea, que faciliten el contacto en tiempo real entre el usuario y la empresa.

- Crear estrategias de promoción enfocadas en los canales directos, como las "ventanas emergentes" con publicidad y uso de "páginas de aterrizaje", que ofrecen incentivos a cambio del registro de nuevos usuarios.

- Crear estrategias de atracción para los nuevos usuarios, como descuentos, regalos u obsequios por la compra realizada a través de la página web.

- Realizar una mejor planificación a nivel estratégico, táctico y operativo de forma física, para poder reflejarla en la extranet e intranet de la empresa.

- Crear un nuevo departamento de mercadeo virtual.

- Realizar un benchmarking de las páginas web de las empresas líderes del sector transporte terrestre y aéreo, ya sean nacionales o internacionales.
- Reestructuración de la página web, para que sea más interactiva y amigable con el usuario, y no solo un portal bancario.

- Creación de una terminal virtual que permita consultar el catálogo de servicios y realizar la compra en línea de tiquetes en la tienda física, como una alternativa a la fila en ventanilla.

- Crear una estrategia de indexabilidad buscando el posicionamiento en el uso eficaz de los motores de búsqueda (SEO, Search Engine Optimization) y la construcción y comercialización de un sitio, con el objetivo de mejorar su posición en los motores de búsqueda (SEM, Search Engine Marketing), y así ser la primera opción en los buscadores para los clientes.

- Mejorar la infraestructura del sitio web, mediante la corrección de las fallas encontradas en el presente estudio.

\section{Copetran}

- Aprovechar su posicionamiento y reconocimiento para subir los precios, con miras a ofrecer mejores servicios en sus rutas más reconocidas.

- Intensificar las estrategias web, para aumentar el grado de interacción de los usuarios por medio de redes sociales, y así tener conocimiento de la opinión de los clientes.

- Creación de una terminal virtual que permita consultar el catálogo de servicios y realizar la compra en línea de tiquetes en la tienda física, como una alternativa a la fila en ventanilla.

- Crear alianzas estratégicas con aerolíneas que compartan las mismas rutas y mantengan un precio asequible para los usuarios, con miras a mejorar los tiempos de viaje.

- Aprovechar su posicionamiento y la adquisición de su pasarela de compra en línea, para estandarizar los precios del mercado.

- Crear estrategias de promoción enfocadas en los canales directos, como las "ventanas emergentes" con publicidad y uso de "páginas de aterrizaje", que ofrecen incentivos a cambio del registro de nuevos usuarios.

- Realizar campañas por redes sociales, correos electrónicos y otros medios publicitarios, para dar a conocer con más intensidad la página web y los 
Katherine Julieth Sierra Suárez, Jessica Tatiana Rey Ordoñez, Angie Patricia Barrios Machado

Análisis de los modelos de negocio en internet: Situación actual en el subsector de transporte intermunicipal de pasajeros en el área

metropolitana de Bucaramanga.

beneficios de comprar por ella.

- Crear una estrategia de indexabilidad buscando el posicionamiento en el uso eficaz de los motores de búsqueda (SEO, Search Engine Optimization), y la construcción y comercialización de un sitio, con el objetivo de mejorar su posición en los motores de búsqueda (SEM, Search Engine Marketing), y así ser la primera opción en los buscadores para los clientes.

- Intensificar las estrategias de precio y promoción, por medio de la página web, con miras a atraer más clientes por este medio.

- Intensificar las estrategias de promoción por medio de redes sociales y página web, con el fin de que los usuarios estén más relacionados con este servicio.

- Aplicar descuentos a los clientes preferenciales que compren desde la página web, sin ocasionar pérdidas para la empresa.

- Para trayectos largos y costosos, establecer mejores servicios al usuario, tales como refrigerios, salas de espera, mejor tecnología, etc. Lo anterior, por medio de un benchmarking del servicio de aerolíneas.

- Crear un espacio de comunicación entre el usuario y la empresa, en tiempo real, más conocido como chat en línea.

- Mejorar la atención a los usuarios en la taquilla y crear un puesto de trabajo que opere en el terminal y la sala VIP que cumpla la función de guía de la página web.

- Aumentar el grado de interacción de los usuarios, por medio de redes sociales y de landing pages de campañas virtuales.

\section{Cotaxi}

- Aprovechar el reconocimiento de la empresa y los canales virtuales existentes, para subir los precios de acuerdo con las temporadas del mercado.

- Crear en su página web un espacio destinado a proveedores y otro espacio para clientes frecuentes, de forma que estos puedan obtener beneficios por usar su plataforma digital.

- Adquirir su propia plataforma virtual de venta de tiquetes, para distinguirse de su competencia, dado que tienen una estructura web similar.
- Obtener un mayor espacio de venta en el terminal de transporte, para ser más visible por el usuario.

- Fortalecer la información suministrada al cliente por medio de la página web y redes sociales, para minimizar los costos en servicio al cliente de forma presencial.

- Realizar campañas virtuales por medio de mailing, de acuerdo con las rutas y horarios más frecuentes de sus clientes, y así mantener al usuario actualizado con dicha información.

- Creación de una aplicación móvil que facilite las rutas y horarios, de modo que el usuario tenga una mayor accesibilidad por medios virtuales.

- Creación de una terminal virtual que permita consultar el catálogo de servicios y realizar la compra en línea de tiquetes en la tienda física, como una alternativa a la fila en ventanilla.

- Crear un departamento de marketing digital enfocado en aumentar la interacción del usuario y el atractivo de la página web.

- Crear alianzas estratégicas con proveedores que cuenten con modelos de negocios en internet, para mejorar la comunicación y reducir costos administrativos.

- Realizar un benchmarking de las páginas web de las empresas líderes del sector transporte terrestre y aéreo, ya sean nacionales o internacionales.

- Reestructuración de la página web, para que sea más interactiva y amigable con el usuario y deje de ser solo un portal bancario.

- Fortalecer la infraestructura de la organización con un plan de adquisición de flotas, a fin de actualizarla tecnológicamente y satisfacer las necesidades de los usuarios.

- Realizar inspecciones periódicas de los vehículos y crear conciencia corporativa en los conductores, para mejorar el servicio prestado.

- Crear una línea de primera clase con servicios especiales en venta de pasajes, atención, comodidad durante la espera y el viaje, diversidad de ofertas, etc.

- Realizar una reestructuración de la estrategia de precio, de tal forma que los usuarios tengan mayores beneficios con la compra en línea. 


\section{Conclusiones}

Se puede concluir que la situación actual de las empresas del subsector de transporte intermunicipal de pasajeros en el área metropolitana de Bucaramanga se encuentra en proceso de exploración e incursión de sus modelos de negocio en internet, con miras a generar desde este medio una ventaja competitiva frente a la competencia.

Las empresas estudiadas son de las más reconocidas en el área metropolitana de Bucaramanga; sin embargo, presentan falencias en su planeación estratégica, no conocen claramente su ventaja competitiva y, por tanto, no pueden reflejarla en su página web.

Las páginas web de las tres empresas analizadas brindan información valiosa a sus clientes y permiten la venta de tiquetes por internet, lo que muestra el esfuerzo que han realizado estas empresas por actualizarse y estar a la vanguardia tecnológica. No obstante, se identifican oportunidades de mejora tendientes a interactuar más con los clientes a través del sitio web, brindarles información de interés, mostrar su ventaja competitiva y cambiar estrategias de precio. De esta forma, aumentan precios de tiquetes hasta donde lo permita la demanda, y se crean ofertas para tiquetes comprados a través de internet.

\section{Agradecimientos}

Especial agradecimiento a la Universidad de Investigación y Desarrollo, por el apoyo brindado en el desarrollo de la investigación, y a los docentes de la Facultad de Ingeniería Industrial, por el tiempo y los conocimientos brindados. Resaltamos el trabajo de la docente Katherine Sierra, por su dedicación y orientación en la investigación.

\section{Referencias}

Alfonzo, P. L., \& Mariño, S. I. (2013). Propuesta de un índice de evaluación web para la estimación de la calidad de sitios web bancarios que operan en la república argentina. Revista GTI, 12(32), 15-32.

Alonso Ferreras, V. H. (2009). Un modelo integral para evaluar la competitividad de destinos turísticos basado en la identificación e integración de factores críticos de éxito. (Doctoral dissertation, Tesis doctoral inédita). Universidad de Las Palmas de Gran Canaria, España. Recuperado de https://accedacris.ulpgc.es/ bitstream/10553/5515/2/0628686_00000_0000.pdf

Botello Peñaloza, H. A., Pedraza Avella, A. C., \& Contreras Pacheco, O. E. (2015). Análisis empresarial de la influencia de las TIC en el desempeño de las empresas de servicios en Colombia. Revista Virtual Universidad
Católica Del Norte, 2(45), 3-15.

Cámara Comercio de Bucaramanga. (2019). Movimiento de pasajeros desde el Terminal de transporte. Recuperado el 12 de marzo de 2019, de Cámara Comercio de Bucaramanga: https://www. camaradirecta.com/temas/indicadoresantander/ indicadores/terminal2018.htm

Cámara de Comercio de Bucaramanga. (2018). Indicadores económicos. Bucaramanga: Cámara de Comercio de Bucaramanga. Recuperado el 2018 de marzo de 12, de http://www.compite360.com/compitehtml5/sitio/ adn_g/

Cámara de Comercio de Bucaramanga. (12 de Marzo de 2019). Movimiento de pasajeros y carga Aeropuerto Palonegro. Recuperado de Cámara de Comercio de Bucaramanga: https://www.camaradirecta.com/ temas/indicadoresantander/indicadores/palo2018. $\mathrm{htm}$

Campdesuñer, R. P., De Miguel Guzmán, M., \& Almaguer, Y. P. (2012). Evaluación de la calidad de los sitios web que comercializan destinos turísticos Cubanos. Estudios y Perspectivas En Turismo, 21(5), 1281-1298. Recuperado de http://www.estudiosenturismo.com. ar/PDF/V21/N05/v21n5a12.pdf

Díaz, R. A. (2014). Marketing y las nuevas tecnologías de información, aplicadas en los negocios. I+ $D$ Revista de Investigaciones, 4(2), 34-48. https://doi. org/10.33304/revinv.v04n2-2014004

Hoque, F. (2000). e-Enterprise: business models, architecture, and components. Londres: Cambridge University Press.

Gürel, E., \& Tat, M. (2017). Swot analysis: a theoretical review. Journal of International Social Research, 10(51), 994-1006.

Khurana, H. E. (2011). E-Commerce: role of e-commerce in today's business. International Journal of Business Management Research, 1(7), 454-461.

$\mathrm{Ma}, \mathrm{H}$. (1999). Creation and preemption for competitive advantage. Management Decision, 37(3), 259-267.

Martínez Rodríguez, E. (2015). El modelo de negocio como base del éxito empresarial: una revisión teórica. Universidad de Almería. Recuperado de http://repositorio.ual.es/bitstream/ handle/10835/3662/3537_EL MODELO DE NEGOCIO COMO BASE DEL EXITO EMPRESARIAL-UNA REVISION TEORICA .pdf? sequence $=1$

Morales Campos, D. R., \&Prieto Daza, M.C. (2017).Sondeo de mercados para la creación de una empresa de asesorías en seguridad vial exclusivamente para transporte terrestre de pasajeros en la sabana de Bogotá. Tesis doctoral. Corporación Universitaria Minuto de Dios. Retrieved from http://repository.uniminuto.edu:8080/ xmlui/bitstream/handle/10656/5523/TEPRO_ MoralesCamposDayany_2017.pdf?sequence=1 
Porter, M. E. (1980). Competitive Strategy: Techniques for analyzing industries and competition. New York: Free Press.

Sánchez, J. I., \& Sandulli, F. (2007). Evolución de los modelos de negocios en internet: situación actual en España de la economía digital. Economía Industrial, 213-229. Recuperado de http://www.minetad.gob.es/Publicaciones/ Publicacionesperiodicas/Economialndustrial/ RevistaEconomialndustrial/364/213.pdf

Terminal de Transportes de Bucaramanga. (2019). Directorio de Empresas. Recuperado el 4 de Abril de 2018, de: http://www.gennessis.com/directorioempresas_ terminal/directorio_empresas.html 